\title{
Desafíos para las transformaciones territoriales en la Región Este del Uruguay
}

Editorial

Adriana Goñi ${ }^{1}$, Isabel Gadino², Mariana Cabrera ${ }^{3}$

El primer número de la Revista Tekoporá está dedicado a relatar algunas de las transformaciones territoriales de la Región Este del Uruguay, espacio que alberga al centro universitario interdisciplinario que da origen a esta mirada integrada entre las Humanidades Ambientales y los Estudios Territoriales. El Centro Universitario Regional del Este (CURE), es parte del proyecto de descentralización de la Universidad de la República, que en los últimos diez años y en conjunto con el gobierno nacional y los departamentales respectivos, ha invertido grandes esfuerzos para equilibrar la centralidad universitaria ubicada en la capital del país, Montevideo. Esto ha permitido la apertura de nuevas carreras de matriz interdisciplinaria, inaugurando diversas sedes que potencian la radicación de la población en los territorios, en particular los jóvenes, habilitando así el desarrollo de capacidades y espacios para que las comunidades situadas puedan repensarse en el contexto nacional e internacional actual.

${ }^{1}$ Centro Universitario Regional Este - UDeLaR / Departamento de Ambiente, Territorio y Paisaje. gondama@gmail.com

2 PDU Grupo de Estudios Territoriales. Centro Universitario Regional del Este - UdelaR Isabelgadino@gmail.com

${ }^{3}$ Centro Universitario Regional Este - UdelaR. m.cabrera.dacosta@ gmail.com 
Los artículos que presentaremos a continuación son una selección de los trabajos expuestos en Viento del Este, ler Coloquio -Debate público sobre transformaciones territoriales en la Región Este. Este evento fue desarrollado en el 2017 y coordinado por las autoras de este texto. El mismo pretendió generar un espacio de difusión de los estudios académicos sobre la Región Este realizados en los últimos años. Por otro lado, se trabajó en la apertura de un espacio para el debate público y la reflexión de las transformaciones territoriales junto a la sociedad civil, actores privados y quienes desearan acercarse a este ámbito.

Los proponentes del Coloquio proveníamos de una multiplicidad de espacios disciplinarios e interdisciplinarios del CURE: el Departamento Territorio Ambiente y Paisaje (DTAP) y el Departamento de Ciencias Sociales y Humanas (DCSH) -los cuales congregan docentes provenientes del Grupo de Estudios Territoriales (GET), el Grupo de estudios sobre migración interna (DCSH), la Licenciatura en Gestión Ambiental, la Licenciatura en Diseño de Paisaje, los Ciclos Iniciales Optativos Social y de Ciencia y Tecnología, la Maestría en Manejo Costero Integrado y la Licenciatura en Turismo.

El encuentro se organizó con exposiciones en cinco principales ejes: Planificación Territorial y Gobernanza, Turismo y desarrollo local, Puesta en Valor de Recursos Naturales y Culturales, Paisaje y Patrimonio, Economías Alternativas y Dinámicas socio- demográficas. Además se realizó un taller final sobre la situación y necesidades de la Región, muestras de Posters, Fotografías, Documentales y Cortos, y se contó con prestigiosos invitados internacionales.

Los resultados obtenidos fueron los de compartir el conocimiento científico con el conjunto de fuerzas vivas de la Región, reforzar las redes de reflexión y acción sobre el futuro y promover la construcción colectiva del territorio regional.

\section{MIRADAS TRASNDISCIPLINARIAS EN LA GESTIÓN DE LOS BIENES COMUNES}

Partimos del paradigma de la complejidad, en donde el territorio es entendido como el producto de la relación entre determinado medio ambiente y los modelos de desarrollo de las culturas que históricamente lo habitaron. Es al mismo tiempo un espacio vivido y normado (Ther, 2012). En este sentido, este número pretende colaborar a comprender los factores e interconexiones que intervienen en la construcción del territorio como sistema socioecológico, incluyendo sus diversas dimensiones, escalas espaciales y temporales, así como los fenómenos sociales y sus consecuencias en el ambiente y el paisaje.

En el primer artículo Tom Angotti, "Justicia ambiental y gestión de los bienes comunes", nos invita a pensar en un largo período, en como los fenómenos que observamos en el territorio de América Latina en la actualidad, pertenecen a procesos históricos que han constituido una organización social y territorial que tiende a concentrarse en lo urbano dando las espaldas al resto de los bienes comunes naturales de nuestro continente. Esto significa que las causas de las actuales crisis ecológicas, en particular la climática, apunta él, son consecuencia de fenómenos de mala gestión de los bienes comunes, en beneficio de determinados grupos sociales, que determinan en este momento la necesidad impostergable de abordar el tema de la Justicia Ambiental. 
La Región Este, objeto de este número, se delimita recientemente como área de actuación de interés estratégico para las políticas públicas, si bien no existe una definición definitiva sobre qué departamentos la conformarían, visto que las Estrategias Regionales de Ordenamiento Territorial de la Región Este (EROT-RE), impulsadas por el Ministerio de Vivienda, Ordenamiento Territorial y Medio Ambiente (MVOTMA), elaboradas en el 2011 y aprobadas en el 2013, incluyen cinco departamentos: Maldonado, Rocha, Treinta y Tres, Lavalleja y Cerro Largo. Mientras que, por otro lado, el Plan Estratégico de Desarrollo de la Región Este (PDR- 2012) impulsado por la Oficina de Planeamiento y Presupuesto a través de su oficina-Uruguay Integra, considera afinidades posibles, en los primero cuatro, excluyendo el departamento de Cerro Largo. acción.

Asimismo, el ámbito académico regional se ha desarrollado en múltiples niveles de

Por un lado, se encuentra el CURE, aprobado en 2006 e integrado por las Sedes de Maldonado, Rocha y la Casa Universitaria de Treinta y Tres. Los ejes prioritarios de este centro son: medio ambiente, biodiversidad, ecología; costa y pesca; desarrollo agropecuario, turismo y minería. Más adelante se inaugura la Casa de la Universidad de Cerro Largo también como parte del proceso de descentralización de la UDELAR. En el documento "Programas Regionales de Enseñanza Terciaria 2008-2010 y su proyección al 2020", se reconoce un gran avance de los últimos años en la construcción de un Sistema Nacional de Enseñanza Terciaria Pública, del cual son en gran medida protagonistas los centros de la regional este. El proceso de consolidar una regional universitaria se construye también con el trabajo cotidiano de sus docentes y alumnado, quienes deben pensarse como parte de una región entendiéndose en primer lugar como Centro Universitario por sede. Esto se hace evidente cuando se observan las colaboraciones en investigación recientes, se vislumbran las temáticas afines, con competencias de enseñanza complementarias, radicadas en los distintos centros, así como egresados que inician actividades profesionales en los temas antes citados.

Las Estrategias Regionales de Ordenamiento Territorial -Región Este, definen un conjunto de desafíos amplios para el territorio en ámbitos de minería, forestación, intensificación de la agricultura (en particular la arrocera, pero también de soja), emprendimientos energéticos, agropecuarios, servicios logísticos y portuarios, sin olvidar el desarrollo del espacio marino con la regulación de las actividades pesqueras, de transporte, explotación de yacimientos, entre otros.

Sin embargo, los fenómenos que atraviesan a la Región Este en la actualidad, nos presentan procesos fermentales, de afirmación y transformaciones de las identidades locales como nos relata el artículo "Mapa multimedia de bienes culturales y de la memoria de la península de Punta Del Este", de Gabriela Campodónico y otros, en los que a través de un trabajo de extensión universitaria se cataliza esa demanda de reforzar las identidades locales, con un crecimiento demográfico importante, pero que solicita recuperar y mapear la memoria y las prácticas culturales a través de instrumentos multimediales. 0 el artículo de Alejandro Folga Relevamientos de espacios exteriores en la ciudad de Maldonado, que a través de un ejercicio pedagógico en la Licenciatura de Diseño de Paisaje releva y realiza un registro gráfico de espacios exteriores que forman parte de edificios públicos vinculados a la cultura, en la ciudad de Maldonado. 
Por otro lado, las transformaciones tienen una contracara no siempre positiva en los social, con procesos de transformación de la matriz productiva en la que la parte más débil de la cadena, en este caso los trabajadores rurales, son los que sufren como describe el artículo de Nicolas Frank, "Espacio, tiempo y necesidades cotidianas. Herramientas de análisis y una mirada a la región Este de Uruguay", una modificación en su espacialidad, en su movilidad y posibilidades reales de acceso a servicios, que hacen difícil imaginar la permanencia de la población en estas áreas.

En los ámbitos de la gestión hay ejemplos de trabajos conjuntos entre gobiernos departamentales y Academia, así como de desencuentros esperables entre ambas posiciones. Para el caso de Rocha, son reconocidos los estudios e iniciativas llevados adelante por el gobierno local, como por ejemplo la zonificación de PROBIDES en los '90, -aunque existen fuertes debates sobre las modificaciones post aprobación (De Álava, 2006; Gadino et al, 2012)la Ordenanza Costera del 2003, que han logrado tutelar algunas áreas de gran valor ecosistémico. Gran parte de la información sobre los ambientes naturales que conforman la región tiene origen en los valiosos trabajos de Probides, los que señalan este territorio como reservorio de recursos únicos o fundamentales a nivel nacional: suelos fértiles, yacimientos geológicos de alto valor, reservas de agua dulce y de biodiversidad y paisajes naturales preciados para el eco-turismo y la conservación (Probides, 1999). Esto se traduce desde la aprobación de la Ley de Ordenamiento Territorial y Desarrollo Sostenible (LOTDS, 2008) en una gradual construcción de Planes Locales, la aprobación de Áreas Protegidas y de sus Planes de Manejo, así como de sus Comisiones de Seguimiento, en forma constante e incremental. En este número presentamos la experiencia de Planificación Colaborativa de los lineamientos de un posible Plan Director para el Parque de Santa Teresa, que nos interrogan sobre quiénes son los actores, que escalas se necesitan en la planificación, y como hacer sinergía entre gobierno y pobladores para buscar formas sostenibles de desarrollo del turismo de naturaleza.

Maldonado también cuenta con un proceso mixto de planificación de su territorio, sin embargo gran parte del desarrollo conceptual y de investigaciones específicas realizadas por la Universidad en la definición de micro regiones y propuestas de planes locales o especiales (Acuña et al, 2006; Acuña et al, 2013 ), no se tradujo en acciones políticas consecuentes, en parte por su no llegada a la ciudadanía y a otros actores que pudieran acompañar estas estrategias y llevarlas adelante en el tiempo. Esto queda claro, cuando se consultan investigaciones recientes que reven las propuestas de desarrollo territorial de esos años, por ejemplo, el circuito rural de Identidad y paisaje Arco del Sol para promover una diversificación productiva en lo turístico y rural. Dichos análisis demuestran que no existieron inversiones particulares ni Planes aprobados que reforzaran el subsistema de pequeñas localidades para esos "Pueblos Invisibles", como los denomina la investigadora Eleonora Leitch, en su artículo "Pueblos (In)Visibles de la Región Este del Uruguay". Del aislamiento a la inclusión socioterritorial de localidades postergadas En Maldonado, Rocha Y Lavalleja. La investigación se amplía gracias al curso guiado por la investigadora con la participación de estudiantes nacionales e internacionales. En este número se pública el artículo Patrimonio cultural y paisaje en la siembra del arroz. Ciudad de Lascano, capital del arroz, de Antonio Moleón (España), Joana Batel (Portugal) - Diego Palau (Uruguay). 
Con una mirada más amplia, los estudios sobre el sistema nacional de ciudades (Altman, Martínez et al, 2015) indican una alta dinámica demográfica para el sur de la Región Este comparado con el resto del país en los últimos años. Se verifica la tendencia de urbanización costera con la conformación del corredor urbano que se está creando entre Montevideo y la aglomeración urbana Maldonado- Punta del Este - San Carlos. Esto nos obliga a preguntarnos desde el punto de vista conceptual y metodológico, qué tipo de modelo de urbanización residencial y turístico desea promover el Uruguay para su litoral Sur, en este caso en particular para su costa atlántica (Medina et al. 2018).

En algunas ocasiones se ve un retroceso en los esfuerzos de organizar y normar el crecimiento del territorio hacia un equilibrio entre ambiente y sociedad. Como nos introduce Javier Yáñez en su artículo Estrategias de acceso al hábitat, instrumentos de gestión urbana y gobiernos locales, las provincias argentinas, con una densidad y flujos mayores de crecimiento, ya han experimentado la dificultad de gobernar las transformaciones territoriales, debido a las diversas escalas e intereses económicos que atraviesan América Latina en la actualidad.

Si bien es aún incipiente a nivel de investigaciones, el estudio de la política de excepciones a la normativa, exoneración tributaria y flexibilización urbana para favorecer la inversión inmobiliaria en Maldonado, nos muestra un avance considerable en Uruguay, de estos mismos intereses económicos internacionales que transforman el territorio. El artículo de Sciandro y Zeballos "Excepciones como instrumento de planificación en Maldonado" plantea una primera aproximación a esta temática, evidenciando que si bien siempre existieron diferencias en las propuestas de zonificación que se realizaron desde la academia, con las promovidas por el Gobierno Departamental, esta política desconoce y debilita las recomendaciones en OT sobre redistribución de cargas y beneficios, tutela de los ecosistemas y paisajes costeros, restando importancia a posibles formas de ocupación sostenible, turismo responsable y acceso justo a servicios ecosistémicos.

Asociado a estas presiones inmobiliarias, vemos que Maldonado se destaca muy ampliamente del resto de los departamentos del país en un acelerado crecimiento demográfico en el último período intercensal (INE, 2004-2011), básicamente a raíz de migraciones desde otros departamentos y cuenta con la segunda aglomeración urbana del Uruguay (Acuña et al, 2013).

Actualmente Maldonado compite con Montevideo como polo de atracción, sobre todo para quienes migran desde los departamentos del Este y Noreste del país. Los movimientos migratorios se concentran principalmente en el área de influencia del eje Maldonado-Punta del Este, territorio atractor no sólo por la expectativa de oportunidades de empleo sino por mejor acceso a servicios (educación, salud, infraestructura, seguridad), factores culturales (propensión a vivir en espacios costeros, búsqueda de calidad de vida, "mística" de Punta del Este como centro turístico, etc.) (Veiga, 2012). Por otra parte, el proceso migratorio se refuerza a sí mismo en base a la constitución de redes de estímulo y apoyo entre quienes han arribado y los potenciales nuevos migrantes del mismo lugar de origen.

La migración provoca efectos en diversas dimensiones tanto para quien migra como para la sociedad de origen y la de destino, como señala Ana Lafourcade en su artículo 
"Migración y espacio social en Maldonado". Más allá de la heterogeneidad causal y el perfil de los migrantes, el proceso migratorio genera quiebres en cuanto al espacio de vida, a los vínculos con el entorno y a las diversas redes en las que participan los individuos en su vida cotidiana. Este quiebre puede ser más o menos agudo, puede estar amortiguado en mayor o menor medida según las posibilidades de inserción y revinculación en la sociedad receptora. La población migrante -sobre todo en el período inmediato al movimiento- puede presentar un déficit importante en el acceso a los diversos servicios y bienes materiales y simbólicos, tales como espacios de socialización, educación, comunicación, recreación, deportes, salud, trabajo y seguridad social (Gerber 2016). Un aspecto de especial relevancia y dificultad es el acceso a la vivienda, sobre todo cuando la migración involucra a varios miembros de una familia. Para la población migrante este proceso genera tensiones entre oportunidades y riesgos (UNESCO, 2012; Rodriguez, 2004). También tiene efectos en la sociedad receptora y en las instituciones públicas y organizaciones del lugar de destino, especialmente cuando la inmigración es cuantitativamente importante y persistente, como sucede en Maldonado. En este caso, la inmigración se hace "visible" por su incidencia en las configuraciones de la sociedad receptora, no sólo en términos demográficos sino sobre todo en la configuración del territorio en aspectos sociales, culturales, económicos y políticos.

Este crecimiento demográfico tiene un fuerte relacionamiento con el desarrollo turístico e industrias asociadas desde el origen de este sector económico en el país como la construcción (Trochon, 2017). Sin embargo, la alta dependencia hacia el turismo internacional deja a la economía local muy vulnerable frente a situaciones que superan la coyuntura del país, generando ciclos muy dispares entre temporadas (Brida et al, 2016). Siguiendo pautas actuales del turismo global en cuanto a servicios ofrecidos (Aledo, 2008; Demajorovik et al, 2011) se están incorporando nuevos desarrollos como son los relacionados al turismo rural y grandes extensiones de complejos cerrados con servicios, incluyendo lagos artificiales y parques de golf. Estas modalidades, en menor medida, también se extienden por la costa oceánica de Rocha, aunque son más recientes y aún no tienen impactos tan significativos.

La apuesta al turismo de altos ingresos que caracteriza históricamente Punta del Este y sigue creciendo en grandes superficies del territorio cercano, básicamente vinculadas a la costa y serranías, conlleva importantes consecuencias de fragmentación socioespacial. Éstas se expresan en un quiebre entre la ciudad estacional -con importantes inversiones tanto públicas en infraestructura y servicios, como privadas en espacios de diversa índole destinados al turismo de élite- y la ciudad permanente, cada vez más extendida en condiciones que van desde lo básico a lo precario (Campodónico, 2008; Acuña et al, 2013, Barindelli et al, 2014).

Con respecto al cuidado de los recursos ambientales y paisajísticos que son los principales atractores del turismo, ambas extensiones residenciales (la turística y la permanente) fomentan la invasión y depredación de espacios críticos para la sostenibilidad de los recursos, como la costa, los humedales, las riberas de arroyos y cañadas y las cumbres de serranías (Probides, 1999; Scasso, 2002; De Álava, 2006; Steffen et al, 2010; Gadino et al, 2012; Acuña et al, 2013). En paralelo, estudios arqueológicos y antropológicos permiten inferir que precisamente estos sitios son potenciales reservorios de restos de culturas precolombinas, generando una alerta sobre el uso de los mismos sin estudios previos a su transformación (Inda et al, 2011; Sotelo, 2014; del Puerto, 2015) 
Vinculado al espacio rural, el análisis de los países de procedencia de los grandes inversores en la Región, arroja datos que estarían alertando sobre el acaparamiento de tierras por parte de capitales extranjeros en desarrollos agropecuarios o la incipiente minería de gran porte (Piñeiro, 2012). Los departamentos de Cerro Largo y Treinta y Tres tienen sus directrices aprobadas, y abordan con Planes Locales como los de la Laguna Merín, o la declaración de área protegida de la Quebrada de los Cuervos, territorios especiales con proyectos definidos. Asimismo los planes locales para sus áreas urbanas y las microregiones asociadas a ellas en elaboración, pueden ser una oportunidad interesante para observar el potencial en estos departamentos.

La Región Este cuenta con una riqueza significativa para estos análisis, en cuanto a características ambientales y paisajísticas (Instituto de Diseño, 2014; CSIC, DTAP, 2016) y puede considerarse un laboratorio experimental en una escala reducida de tiempo y espacio, de cambios contundentes. El artículo de Cristina Zurbriggen y Mariana Gonzalez, "Innovación en el abordaje de problemas complejos para una transformación sostenible", contribuye desde la teoría a la búsqueda de respuesta de frente a la necesidad de construir herramientas de gobernanza y análisis multifactorial de los procesos de transformación territorial. Como demuestra Hugo Partucci en su artículo "Parece, pero no es lo mismo. El uso de conceptos socialmente compartidos en la comunicación y construcción de conocimiento sobre las sequías en el sureste de Uruguay", estos ámbitos son necesarios porque los lenguajes y puntos de vista de los diversos actores en torno a temas claves, no pueden seguir gestionándose como datos secundarios de la realidad, sino que se necesitan metodologías transdisciplinarias que aborden la construcción colectiva de políticas públicas de frente a transformaciones crecientes que afectan seriamente los modos de producción tradicionales. En la misma línea tanto el artículo de Ingrid Roche y Adriana Goñi Planificación Colaborativa para el Parque de Santa Teresa, Rocha, como el de Micaela Trimble y Ryan Plummer "Evaluación participativa para la gobernanza adaptativa de sistemas socio-ecológicos", nos relatan experiencias exitosas, que demuestra como estas nuevas formas de gobernanza multinivel, con matices y aún mejorables, están siendo demandadas y experimentadas en Uruguay.

Esta número de la Revista Tekoporá y un segundo número que recoge ulteriores artículos del Coloquio Viento del Este, pretenden compartir lo expuesto en el encuentro para promover la circulación de conocimientos, reflexiones e ideas para un análisis crítico del desarrollo territorial y la reconversión sostenible de la Región Este.

Buena lectura! 


\section{REFERENCIAS BIBLIOGRÁFICAS}

Acuña, C. (Responsable Académico), de Souza, L. (Editor), Gadino, I; Leicht, E. (Coordinadora del Equipo Técnico), Musso, C; Vainer, D; Varela; A. Colaboradores: Finozzi, A; Osimani, V; Pastorino, S; Plada, A. 2013. "Aglomeración Maldonado - Punta del Este - San Carlos. Enfoques y propuestas hacia un Modelo transformador.". 120 pp. ISBN 978-9974-99-045-6. Mosca Hnos.

Acuña A., De Souza L, Gadino, I. ,Leicht E, Musso C., Vainer D, Varela A., (2010). Talleres Territoriales Microregionales 0 al 8. Ed Farq. ITU. Udelar

Acuña A., De Souza L, Leicht E, Musso C., Vainer D, Varela A., (2013). Aglomeracion Maldonado, San Carlos, Punta del Este. Ed Farq. ITU. Udelar.

Aledo, A. 2008. "De la tierra al suelo: la transformación del paisaje y el nuevo turismo residencial". En: ARBOR Ciencia, Pensamiento y Cultura

CLXXXIV 729 enero-febrero (2008) 99-113 ISSN: 0210-1963

Altman, L. Martinez, E. (2015) Entre la macrocefalia estructural y el policentrismo emergente Modelos de desarrollo territorial en el Uruguay (1908- 2011), CSIC, FADU, UDELAR.

Aznáres, C. (2017). Distirbución social y valoración de servicios ecosistémicos en el humedal del Arroyo Maldonado. Programa de Apoyo a la Investigación Estudiantil. CURE-LGA- CSIC

Barindelli, N. Gadino, I., Goñi, A.L., Inda, H., Piazza, N., (2011). Nuevas urbanizaciones turísticas en la costa uruguaya. Análisis de la percepción local. Balneario Buenos Aires-La Bota y barrios cerrados aledaños. Documento CURE CSIC.

Barindelli, N, Gadino, I; Goñi, A. L; Migues, P. 2014. "Procesos actuales de construcción del territorio en el municipio de Maldonado. La Faja Pinares - San Jorge". UPE 11 Conducir las Transformaciones Urbanas: un debate sobre direcciones, orientaciones, estrategias y políticas que modelan la ciudad futura / Carlos Andrés Abate... [et.al.]; compilado por Jorge Leonardo Karol... [et.al.]. - 1a ed. - La Plata: Universidad Nacional de La Plata. E-Book.ISBN 978-950-341133-91. Planificación Urbana . I. Abate, Carlos Andrés II. Karol, Jorge Leonardo, comp. CDD 307.12

Brida, J. González, M. N. Lanzilotta, B. Análisis de los Determinantes del Turismo Interno en Uruguay. 2016. REVISTA DE ESTUDIOS REGIONALES № 108, I.S.S.N.: 0213-7585 (2017), PP. 43-78.

Campodónico, R. 2008. "El turismo y los vaivenes del desarrollo". Aportes y Transferencias, 12(1), 15-40. ISSN 0329-2045

De Âlava, D..2006. "Costa uruguaya Interfase de conflictos: el sistema costero de Rocha" (Uruguay). Bases para la Conservación y el Manejo de la Costa Uruguaya: 637-649.

del Puerto, L. 2015. "Interrelaciones humano-ambientales durante el Holoceno tardío en el este del Uruguay: cambio climático y dinámica cultural”. (Tesis Doctorado en Ciencias Biológicas), Facultad de Ciencias, Universidad de la República 
Demajorovic, J; Aledo Tur, A; Landi, B. y Mantovani Kondo A.2011. "Complejos turísticos residenciales - Análisis del crecimiento del turismo residencial en el Mediterráneo español y en el Litoral Nordestino (Brasil) y su impacto socio-ambiental". En: Estud. perspect. tur. vol.20 no.4 Ciudad Autónoma de Buenos Aires jul./ ago. 2011. versión On-line ISSN 1851-1732

Escudero, L.; Oggero, C.; Moreira, M.; Fornaro, J. (2012). Ordenamiento territorial en clave de inteligencia territorial: El caso de Lavalleja, Uruguay. XI INTI International Conference La Plata, Argentina.

Gadino, I; Brazeiro, A; Panario, D; Roche, I; Gutierrez, O. (2012). "El modelo actual de desarrollo turístico al oeste del Balneario La Paloma, Rocha, Uruguay. Tendencias, riesgos y propuestas". Sustentabilidade em Debate, Vol. 3, № 2, 2012. 1/ 7/ 12. PP 21-39. Centro de Desenvolvimento Sustentável - Universidade de Brasília, Brasil. ISSN: ISSN-e 2179-9067. http:/ / seer.bce.unb.br/ index.php/ sust/ article/ view/ 8125/ 6162

Gerber, E. (2016) Significados y usos de la alteridad. Una aproximación etnográfica a los procesos de producción de subjetividad vinculados a la movilidad geográfica en la ciudad de Maldonado. (Tesis inédita de maestría). Universidad de la República, Uruguay.

Goñi Mazzitelli, A. (2011). "Consensus Building; the democracy which works properly in complex society". Entrevista a Lawrence Susskind, Rivista Trama, Journal of Land Use Mobility and Environment, Italy.

Inda, H., del Puerto, L., Bracco, R., Castiñeira, C., Capdepont, I., Gascue, A. and Baeza, J. 2011. "Relación Hombre-Ambiente para la costa estuarina y oceánica del Uruguay durante el Holoceno. Reflexiones y Perspectivas". In: Garcia-Rodríguez, F. (ed.) El Holoceno en la zona costera del Uruguay. 229-260.

Inda, H., Goñi, A.L., Piazza, N. et alt (2017). Transformaciones territoriales y paisajísticas, dinámicas e indicadores. Informe Final Proyectos I+D, Comisión Sectorial de Investigación Científica.

Instituto de Diseño, 2014. "Pautas y recomendaciones para el Ordenamiento Paisajistico del Departamento de Maldonado". ISBN: 978-9974-0-1328-5

Instituto de Diseño. Sommaruga, R. et. al. 2010. "La frontera del agua. El paisaje costero del Uruguay" . Montevideo, Mosca. 256 pág. ISBN: 978.9974-0-0631-7

Instituto Nacional de Estadística. 2012. Resultados del Censo de Población 2011: población, crecimiento y estructura por sexo y edad. Montevideo, INE.

Leitch, E. et alt. (2017). Pueblos (In)visibles de la Región Este del Uruguay. Del aislamiento a la inclusión socio territorial de localidades postergadas en Maldonado, Rocha y Lavalleja. En Ateneos ITU N2, ed FADU.

Medina, M; Piazza, N; Leicht, E; Garat, D. 2008. "Manejo del paisaje como recurso turístico en Uruguay". ISBN 978-9974-0-0439-9 y ISBN 978-9974-0-0440-5 
Medina M.; Altmann, L; Alves, J; Jorge, M; Logiuratto, L; Matos, A; Rabelino, C; Tobler, C.. (2018). Territorios litorales y costeros. Tres casos en la Costa Sur uruguaya, criterios para la valorización de sus paisajes. En Ateneos ITU N3, ed FADU- UDELAR.

Ostrom, E. (1990). Governing the Commons: The Evolution of Institutions for Collective Action, Cambridge University Press.

Piñeiro, D. 2012, "Land grabbing: concentration and "foreignisation" of land in Uruguay" Canadian Journal Of Development Studies / Revue Canadienne D'études Du Développement Vol. 33 , Iss. 4,2012

PROBIDES, 1999. Plan Director. Reserva de Biosfera Bañados del Este / Uruguay.- Rocha, UY. ISBN 9974753236

Rodríguez, A. (2014). Estudios de Cadenas productivas territoriales de valor para promover el desarrollo local con inclusión social. Ed. IECON. Imp Mastergraf.

Rodriguez, Jorge (2004). “Migración Interna en América Latina y el Caribe: estudio regional del período 1980-2000". CEPAL, Serie Población y Desarrollo, Santiago de Chile

Scasso, F. 2002. AMBIENTES ACUÁTICOS DE LA ZONA COSTERA DE LOS HUMEDALES DEL ESTE ESTADO ACTUAL Y ESTRATEGIAS DE GESTIÓN. PROBIDES, Documento de trabajo № 43. 20112013

Soler, S., Sotelo, M. 2014. Màs cerca del cielo. Misterios de la arqueología uruguaya para niños curiosos. Banda Oriental.

Steffen, M; Inda, H. (eds.) 2010. "Bases técnicas para el manejo integrado de Laguna del Sauce y cuenca asociada". Universidad de la República y South American Institute for Resilience and Sustainability Studies (SARAS)

Ther, F (2012). “Antropologia del territorio". Revista Polis № 32

Trochon, Y. 2017. Punta del Este. El edén oriental (1907-1997). Editorial Fin de Siglo. 504 p. ISBN: UNESCO. 2012. "Inclusión de los migrantes en las ciudades. Políticas y prácticas urbanas innovadoras".978-9974-49-866-2.

Veiga, D. (coord) 2012. "Crecimiento Económico y Desigualdad Social en Maldonado". Facultad de Ciencias Sociales, CURE, UDELAR, IDM. Secretaría de Comunicación y Prensa, IDM, Maldonado.

Documentos:

MVOTMA (2008) Ley No 18.308, Ordenamiento Territorial y Desarrollo Sostenible, Marco Regulador General, Uruguay.

MVOTMA, (2013) Estrategias Regionales de Ordenamiento Territorial y Desarrollo Sostenible de la Región Este, Uruguay. 
MVOTMA, (2000) Proyecto de Ordenanza Costera del Departamento de Rocha Dirección de Medio Ambiente Intendencia de Rocha, PROBIDES, PNUD, Unión Europea, Ed. Global Environment Facility.

MVOTMA - DINOT (2018). Análisis de la política de exoneración tributaria y flexibilización urbana en el departamento de Maldonado. Informe. http:/ / www.mirador.cure.edu.uy/ proyecto_ampliado.php?id=85

Intendencia Municipal de Rocha (2003). Decreto del Plan General Municipal de Ordenamiento y Desarrollo Sustentable de la Costa Atlántica del Departamento de Rocha.

Plan Estratégico de Desarrollo de la Región Este, (2012) Oficina de Planificación y Prespuesto, www.agev.opp.gub.uy/observatorio_docs/ publico/ 228.pdf

Probides (1999), Plan Director para la Reserva de Biósfera, Humedales del Este. Programa de conservación de la biodiversidad y desarrollo sustentable en los humedales del este. 